\title{
Erratum: A very dark stellar system lost in Virgo: kinematics and metallicity of SECCO 1 with MUSE
}

\author{
by G. Beccari, ${ }^{1 \star}$ M. Bellazzini, ${ }^{2}$ L. Magrini, ${ }^{3}$ L. Coccato,${ }^{1}$ G. Cresci, ${ }^{3}$ F. Fraternali, ${ }^{4}$ \\ P. T. de Zeeuw, ${ }^{1,5}$ B. Husemann, ${ }^{1}$ R. Ibata,${ }^{6}$ G. Battaglia, ${ }^{7}$ N. Martin,,${ }^{6,8}$ V. Testa, ${ }^{9}$ \\ S. Perina ${ }^{2}$ and M. Correnti ${ }^{10}$ \\ ${ }^{1}$ European Southern Observatory, Karl-Schwarzschild-Strasse 2, D-85748 Garching bei München, Germany \\ ${ }^{2}$ INAF - Osservatorio Astronomico di Bologna, Via Ranzani 1, I-40127 Bologna, Italy \\ ${ }^{3}$ INAF - Osservatorio Astrofisico di Arcetri, Largo E. Fermi 5, I-50125 Firenze, Italy \\ ${ }^{4}$ Dipartimento di Fisica and Astronomia, Università degli Studi di Bologna, Viale Berti Pichat, 6/2, I-40127 Bologna, Italy \\ ${ }^{5}$ Leiden Observatory, Leiden University, Postbus 9513, NL-2300 RA Leiden, the Netherlands \\ ${ }^{6}$ Obs. astronomique de Strasbourg, Université de Strasbourg, CNRS, UMR 7550, 11 rue de l'Université, F-67000 Strasbourg, France \\ ${ }^{7}$ Instituto de Astrofisica de Canarias, E-38205 La Laguna, Tenerife, Spain \\ ${ }^{8}$ Max-Planck-Institut für Astronomie, Königstuhl 17, D-69117 Heidelberg, Germany \\ ${ }^{9}$ INAF - Osservatorio Astronomico di Roma, via Frascati 33, I-00040 Monteporzio, Italy \\ ${ }^{10}$ Space Telescope Science Institute, Baltimore, MD 21218, USA \\ ${ }^{11}$ Universidad de La Laguna, Dipartimento Astrofisica, E-38206 La Laguna, Tenerife, Spain
}

Key words: errata, addenda $-\mathrm{H}$ II regions - galaxies: dwarf - galaxies: star formation.

This paper 'A very dark stellar system lost in Virgo: kinematics and metallicity of SECCO 1 with MUSE' was published in MNRAS, 465, 2189 (2017). While performing a follow-up study of SECCO1, we discovered an unfortunate error in our calculation of the star formation rate (SFR) reported in the original paper. The error turns into an overestimation by a factor of 100 of the SFR. The correct SFR of SECCO1 as inferred from the MUSE data is $0.7 \times 10^{-3} \mathrm{M}_{\odot} \mathrm{yr}^{-1}$, a value that is typical for star-forming dwarfs of similar luminosity.

As a consequence, the following conclusion, reported in Section 6 of the paper 'The extreme SFR, given the total luminosity, may be suggestive of a star formation episode induced by some kind of interaction.' is not valid anymore since the correct SFR reconciles SECCO1 to the class of dwarf galaxies of similar luminosity.

The last sentence in the abstract is now changed as follows:

The star formation rate derived from the $\mathrm{H} \alpha$ is $0.7 \times$ $10^{-3} \mathrm{M}_{\odot} \mathrm{yr}^{-1}$, quite typical of star-forming dwarfs of comparable luminosity.

The last paragraph of Section 5 of the original paper should read as follows:

On the other hand, integrating the extinction-corrected $\mathrm{H} \alpha$ luminosity over all the $38 \mathrm{H}$ II regions and adopting the calibration by
Kennicutt (1998), we obtain a total SFR of $0.7 \times 10^{-3} \mathrm{M}_{\odot} \mathrm{yr}^{-1}$, quite typical of star-forming dwarfs of comparable luminosity $\left(\simeq 1.0 \times 10^{-3} \mathrm{M}_{\odot} \mathrm{yr}^{-1}\right.$; James et al. 2015, see, e.g. their fig. 6).

Moreover, we spotted a typo in the abstract of the original paper. The mean abundance $<12+\log (\mathrm{O} / \mathrm{H})>=8.44$ is in fact $<12+\log (\mathrm{O} / \mathrm{H})>=8.38$, as inferred from the metallicity values of 8.37 and 8.39 of the Main and Secondary Body (respectively) reported in Table 2 of the manuscript. This typo has no consequence on any of our conclusions.

\section{ACKNOWLEDGEMENTS}

We gratefully thank David Sand for spotting and pointing us to the errors in the published manuscript.

\section{REFERENCES}

James B. L., Koposov S., Stark D. P., Belokurov V., Pettini M., Olszewski E. W., 2015, MNRAS, 448, 2687

Kennicutt R. C., Jr, 1998, ARA\&A, 36, 186

This paper has been typeset from a $\mathrm{T}_{\mathrm{E}} \mathrm{X} / \mathrm{LT}_{\mathrm{E}} \mathrm{X}$ file prepared by the author. 